\title{
POTENCIAL PARA OBTENÇÃO DE ÓRGÃOS EM UM HOSPITAL DE URGÊNCIA DE SERGIPE
}

\author{
Potential for Organ procurement potential in an emergency hospital of Sergipe
}

\section{RESUMO}

Emília Cervino Nogueira1, Carlos Umberto Pereira²

Objetivos: Estimar o potencial de doadores de órgãos em um hospital de referência em urgência; descrever seu perfil e analisar as causas da não efetivação da doação. Métodos: Estudo quantitativo, descritivo e retrospectivo utilizando dados do prontuário de pacientes até 65 anos de idade que morreram por traumatismo cranioencefálico, acidente vascular encefálico ou tumor primário do sistema nervoso central nos anos de 2005 a 2007. Resultados: Dos 645 prontuários analisados, 219 tiveram registro sugestivo de morte encefálica, sendo 126 com Termo de Declaração de Morte Encefálica preenchido. A mediana da idade desses potenciais doadores foi 29,5 anos; houve predomínio do sexo masculino (77\%), de vítimas de traumatismo cranioencefálico (68\%) e de internados na Unidade de Urgência (41\%). Metade deles apresentou sinais de morte encefálica nos dois primeiros dias de hospitalização e apenas 7\% se tornaram doadores efetivos, sendo a recusa da família (31\%) e parada cardíaca irreversível (28,6\%) as principais causas da não efetivação da doação. O tempo entre o primeiro registro e a remoção dos órgãos foi maior que 30 horas em $75 \%$ dos doadores. Conclusões: Os resultados do estudo mostraram uma baixa notificação e diagnóstico de morte encefálica, ocasionando um número reduzido de potenciais doadores, e, por conseguinte, um pequeno número de doadores falecidos de órgãos e tecidos. As taxas de recusa da família e a parada cardíaca irreversível sugerem a necessidade de ações educativas dirigidas aos profissionais de saúde e à sociedade em geral.

Descritores: Morte Encefálica; Obtenção de Tecidos e Órgãos; Transplante de Órgãos; Registros Médicos.

\footnotetext{
Instituições:

1 Central de Notificação, Captação e Distribuição de Órgãos de Sergipe - Aracaju, Sergipe, Brasil.

2 Departamento de Medicina da Universidade Federal de Sergipe - Aracaju, Sergipe, Brasil.
}

\section{Correspondência:}

Emília Cervino Nogueira

Avenida Augusto Franco, 2000 - Pq.Resid.Vivendas de Aracaju - Quadra 04 Lote 05

Bairro Siqueira Campos

CEP: 49075-100 - Aracaju - Sergipe - Brasil

Tel/Fax: (79) 3259-3491

E-mail: jienogueira@ig.com.br

Recebido em: 21.08.2007
Aceito em: 13.09.2007

\section{INTRODUÇÃO}

O Brasil destaca-se no cenário mundial de transplantes. Tais procedimentos são realizados neste país há mais de 40 anos, tendo atualmente um dos maiores programas públicos de transplantes do mundo, com 548 estabelecimentos de saúde e 1354 equipes médicas autorizadas pelo Sistema Nacional de Transplantes (SNT). ${ }^{1}$

Sergipe também tem histórico nessa área, sendo o primeiro estado do Norte e Nordeste, sendo o sexto no Brasil a realizar um transplante cardíaco, em $1986 .^{2}$

No entanto, a captação de órgãos para transplante no Brasil ainda é reduzida se comparada com alguns países, ${ }^{3-6}$ e insuficiente se for levada em conta a necessidade. Dados do Ministério da Saúde indicam a existência de quase 70 mil pessoas aguardando por um transplante no país em 2007. Em Sergipe são cerca de 600 pessoas nesta situação. ${ }^{1}$

O grande problema é que a maioria dos transplantes só pode ser realizada com órgãos obtidos de doadores em morte encefálica (ME), ou seja, indivíduos que apresentam cessação completa e irreversível das funções encefálicas, mas que mantêm, ainda que artificialmente e por tempo limitado, os batimentos cardíacos e a circulação sangüínea.?

A Lei 9.434/97, que dispõe sobre a retirada de órgãos, tecidos e partes do corpo humano para fins de transplante remete ao Conselho Federal de Medicina (CFM) a competência de definir o diagnóstico 
de ME e determina a obrigatoriedade de sua notificação pelos estabelecimentos de saúde. ${ }^{8}$

Sendo assim, o CFM, em sua Resolução 1480/97, estabeleceu o protocolo para diagnóstico de $\mathrm{ME}$, no qual devem ser realizados dois exames clínicos neurológicos que identifiquem coma aperceptivo com ausência de atividade motora supra-espinal e apnéia, além de exame complementar que mostre ausência de perfusão sanguínea ou de atividade elétrica ou metabólica cerebral. Os exames devem ser registrados no "Termo de Declaração de Morte Encefálica”. ${ }^{9}$

O Decreto 2268/97 ainda acrescenta que os exames devem ser realizados por dois médicos, no mínimo, um dos quais com título de especialista em Neurologia reconhecido no país. Os médicos integrantes das equipes de captação e transplante autorizadas pelo SNT não podem participar do processo de verificação de ME. ${ }^{10}$

Estudos realizados no país têm demonstrado possíveis falhas na detecção de ME, o que vem comprometendo a identificação dos potenciais doadores e, conseqüentemente, a oferta de órgãos para transplante. ${ }^{11-15}$

Em Sergipe, uma pesquisa em laudos necroscópicos do Instituto Médico Legal em 2002 identificou a existência de baixo aproveitamento de órgãos no estado. ${ }^{16}$

A partir desse contexto, o presente estudo objetivou estimar o potencial de doadores de órgãos em um hospital de referência em urgência e emergência do estado de Sergipe, descrever o seu perfil demográfico e analisar as causas da não efetivação da doação.

\section{MÉTODOS}

Trata-se de um estudo de caráter quantitativo, descritivo e retrospectivo, realizado em um hospital de referência de urgência e emergência do estado de Sergipe.

Foram avaliados os prontuários de pacientes de até 65 anos de idade com diagnóstico de traumatismo cranioencefálico (TCE), acidente vascular encefálico (AVE) ou tumor primário do sistema nervoso central (SNC) sem metástase, no período de janeiro de 2005 a dezembro de 2007.

Esses diagnósticos foram selecionados, uma vez que são as causas mais freqüentes de ME. 17,18 Além disso, considerou-se a restrição de faixa etária por se tratar da idade limítrofe para a doação de órgãos sólidos no estado onde se deu a pesquisa.

Utilizou-se um instrumento de coleta de dados contendo as seguintes variáveis: dados sociodemográficos e epidemiológicos (unidade de internação, idade, sexo e causa do óbito); dias de internação (da admissão até o primeiro registro sugestivo de ME na evolução médica e total de dias de hospitalização); tempo (em horas) entre a suspeita de $\mathrm{ME}$ e a realização do primeiro exame do protocolo de $\mathrm{ME}$ entre cada exame e tempo total desde a suspeita até a remoção dos órgãos ou a parada cardíaca irreversível em doadores e não doadores e causas da não realização dos exames para o diagnóstico de ME, quando fosse o caso.

As causas da não conclusão do protocolo de ME foram obtidas também a partir de registros da Central de Notificação, Captação e Distribuição de Órgãos de Sergipe (CNCDO-SE). A partir dos dados coletados, foram considerados potenciais doadores os pacientes que tinham o Termo de Declaração de Morte Encefálica ${ }^{9}$ com pelo menos o primeiro exame preenchido.

Os dados coletados foram analisados com auxílio dos programas Windows Excel e Statical Package for Social Sciences (SPSS) versão 13.0. As variáveis quantitativas foram descritas a princípio como média e valores mínimo e máximo, contudo, por não assumirem uma distribuição gaussiana, foram apresentadas em mediana e percentagem.

O estudo foi aprovado pelo Comitê de Ética em Pesquisa envolvendo seres humanos da Universidade Federal de Sergipe sob o número CAAE - 0978.0.000.107-07 e constitui-se parte da dissertação de Mestrado da autora responsável.

\section{RESULTADOS}

No período estudado ocorreram 645 óbitos de pacientes com até 65 anos de idade devido a TCE, AVE ou tumor primário do SNC sem metástase. A prevalência de óbitos com registro sugestivo de ME foi de 34\% (219/645).

No entanto, 43\% destes (93/219) nem sequer iniciaram o protocolo de $\mathrm{ME}$ e em apenas 5\% (11/219) o processo foi completado. A distribuição dos pacientes conforme a realização dos exames é apresentada na Figura 1.

Figura 1: Distribuição dos pacientes com registro sugestivo de morte encefálica, conforme realização dos exames do protocolo para o diagnóstico $(N=219)$.



As causas para a não realização dos exames nos pacientes cujo protocolo de ME não foi aberto não foram registradas em $83 \%$ (77/93) dos casos. Em sete pacientes, condições clínicas como hipotermia e hipotensão inviabilizaram a realização do exame clínico. Seis pacientes estavam em uso de droga depressora do SNC e não houve suspensão de sua prescrição, de forma que o protocolo de ME não foi iniciado. Um paciente fez parada cardíaca irreversível antes da realização do primeiro exame (Figura 2).

Figura 2: Causas da não-realização do protocolo para diagnóstico de morte encefálica $(N=93)$.

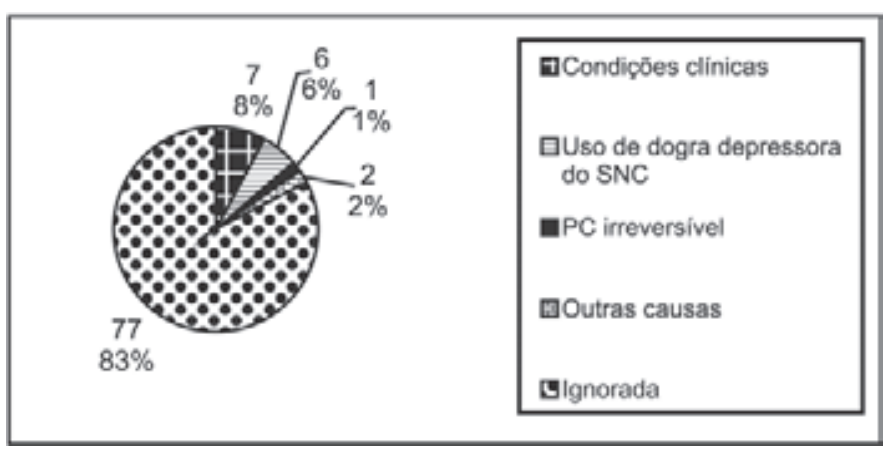


Foram considerados potenciais doadores 126 pacientes com pelo menos o primeiro exame do protocolo de ME. A mediana da idade desses pacientes foi de 29,5 anos, sendo que 75\% deles tinham menos de 50 anos. Predominaram pacientes do sexo masculino, com 77\% (97/126) dos casos.

A causa de óbito mais comum foi TCE com 68,3\% (86/126), seguida de AVE com 28,6\% (36/126), sendo tumor primário de SNC apenas 3,1\% (4/126).

Com relação à unidade de internação em que esses pacientes se encontravam, observou-se maior incidência na urgência (41\%), seguida da Unidade de Terapia Intensiva - UTI (25\%) e da Unidade de Recuperação Anestésica - RPA (22\%). Faz-se necessário esclarecer que a unidade semi-intensiva, que teve $10 \%$ dos potenciais doadores, só começou a funcionar a partir de 2007. Apenas 2\% se encontravam em enfermaria.

Ao analisar o tempo decorrido entre a admissão no hospital e a suspeita de ME, observou-se mediana de um dia, sendo que $25 \%$ dos pacientes apresentaram registro sugestivo de ME já nas primeiras horas após a admissão. A mediana do tempo total de internação foi de quatro dias, sendo o P 25 de três dias e o P 75 de sete dias.

Com relação ao tempo para a realização dos exames do protocolo de ME, observou-se que metade dos pacientes ( $\mathrm{P}$ 50) teve mais de 17 horas entre a suspeita de ME e o início do protocolo. Apenas $10 \%$ dos potenciais doadores tiveram um intervalo de seis horas entre o primeiro e o segundo exames; em metade deles o intervalo foi maior que 12 horas e em 25\% passou das 20 horas.

Dos 11 pacientes que tiveram protocolo completo, $50 \%$ fizeram exame complementar mais de 15 horas após o segundo exame clínico.

A mediana do tempo total desde a suspeita até o término do processo com a conseqüente remoção dos órgãos entre os doadores (9 pacientes) foi de 37,5 horas.

Metade daqueles potenciais doadores cuja doação não foi efetivada devido à recusa familiar, sorologia reagente ou outras causas $(90$ pacientes) permaneceram mais de 70 horas desde o primeiro registro de ME até a parada cardíaca irreversível. Já a mediana do tempo total desde a suspeita até a interrupção do processo entre aqueles que tiveram protocolo de ME aberto, mas não completado devido à parada cardíaca irreversível (36 pacientes) foi de 37 horas.

Entre os potenciais doadores, foram realizados dois exames clínicos do protocolo em 71 (56,3\%) pacientes, enquanto 21 (16,7\%) tiveram o processo interrompido após o primeiro exame, devido à parada cardíaca irreversível. Observou-se que existe ligação entra a realização do protocolo de ME e a doação de órgãos, já que em 13 casos (sete por recusa familiar para a doação e seis com sorologia reagente) o processo não teve continuidade, uma vez que a remoção dos órgãos foi descartada. Apenas 11 (9\%) potenciais doadores tiveram o protocolo de ME completado. Mais uma vez, é clara a ligação entre o diagnóstico de ME e a doação de órgãos para transplante: 35 pacientes $(27,8 \%)$ tiveram como causa da não realização de todos os exames do protocolo a recusa da família para a doação, 15 (11,9\%) sorologia reagente para doenças infecto-contagiosas e 11 (8,7\%) por não serem considerados "bons" doadores devido às condições clínicas (Figura 3).

As causas predominantes para a não remoção dos órgãos foram: recusa da família em 31\% dos casos (39/126) e parada cardíaca irreversível em 28,6\% (36/126). Ao final, dos 126 potenciais doadores, apenas nove (7\%) tornaram-se doadores efetivos.
Figura 3: Distribuição de potenciais doadores, conforme causas da não-realização dos exames de protocolo para diagnóstico de morte encefálica. $N=126$

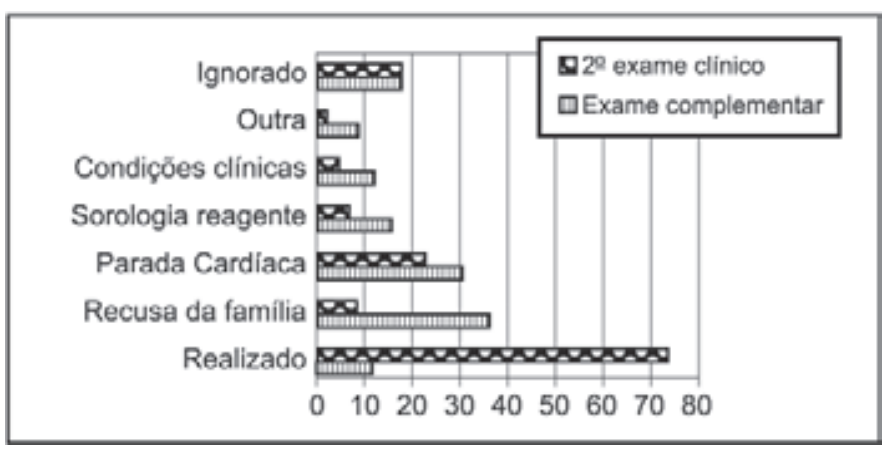

\section{DISCUSSÃO}

É amplamente conhecido que a obtenção de órgãos no Brasil, como de resto em todo o mundo, tem sido insuficiente para suprir a demanda de receptores que necessitam de transplante, tendo em vista as listas que crescem a cada dia. Os avanços científicos e tecnológicos na área de transplante, que têm permitido a realização de procedimentos com excelentes resultados, esbarram na escassez de doadores. Em Sergipe, os registros estatísticos da Central de Notificação, Captação e Distribuição de Órgãos - CNCDO informam que houve 116 notificações de potenciais doadores nos anos 2005 a 2007 em todos os hospitais do estado, a maioria obtida por busca ativa, dos quais apenas nove se tornaram doadores efetivos. No presente estudo encontrou-se 126 potenciais doadores no mesmo período somente em um hospital, conquanto seja o de referência de urgência e emergência no estado. Se forem acrescentados aqueles com registro sugestivo de ME na evolução médica, mas sem preenchimento do Termo de Declaração de Morte Encefálica, o número sobe para 219.

Esse achado demonstra claramente a existência de subnotificação. Estudo realizado em São Paulo, maior centro de transplantes no país, revela que apenas um quinto dos potenciais doadores é notificado às CNCDO, comprometendo seriamente a identificação de possíveis doadores e sua conversão em doadores efetivos. ${ }^{11} \mathrm{Na}$ Europa, à exceção da Espanha, onde os índices de doação estão entre os mais elevados do mundo, a realidade não tem sido muito diferente. ${ }^{19,20}$

Tão baixa notificação talvez se deva, além do desinteresse, ao desconhecimento por parte dos profissionais da obrigatoriedade determinada por lei. ${ }^{8,9}$ Uma pesquisa com profissionais de UTI revelou que $27 \%$ dos médicos e $34 \%$ dos enfermeiros desconheciam ser a ME de notificação compulsória; metade deles não se sentia responsável por sua comunicação e apenas 25\% já haviam feito uma notificação durante sua rotina de trabalho. ${ }^{21}$

Dos 219 pacientes com registro sugestivo de ME detectados neste estudo, 93 (43\%) não iniciaram o protocolo de ME para diagnóstico, conforme determina a legislação. ${ }^{9}$

Mais preocupante foi a constatação de que apenas 5\% tiveram o protocolo completo, ou seja, os dois exames clínicos e o

* Dados fornecidos pela Central de Notificação, Captação e distribuição de Órgãos de Sergipe em 03/04/2008. 
complementar. Esse índice difere dos relatados de outros estudos no país: um levantamento realizado em sete UTI pediátricas nas diversas regiões do Brasil mostrou que em $80 \%$ dos casos o diagnóstico foi completado, sendo que no Nordeste o índice foi de apenas $72 \%{ }^{12}$ É possível que essa diferença ocorra pelo fato do referido levantamento ter sido realizado somente em UTI, enquanto o presente estudo contemplou todas as unidades do hospital. No entanto, um outro estudo em uma Organização de Procura de Órgãos - OPO de São Paulo revelou que o diagnóstico não foi concluído em 6,25\% dos pacientes. ${ }^{22}$ A falta de conclusão no diagnóstico pode refletir a dificuldade de estrutura para a realização do exame complementar, mas também pode estar relacionada a problemas médicos. Um levantamento conduzido pela Associação Brasileira de Transplante de Órgãos (ABTO) com profissionais especialistas em transplantes no âmbito nacional revelou que no Nordeste, $63 \%$ dos profissionais referem dificuldades para confirmação do diagnóstico de $\mathrm{ME}^{23}$

Ao serem analisadas as causas para a não conclusão do protocolo de ME, observa-se que $49 \%$ dos potenciais doadores não tiveram todos os exames realizados por motivos relacionados à doação de órgãos. Na pesquisa realizada em UTI pediátricas brasileiras, os autores sugerem que o protocolo de ME pode não ter sido completado pelo fato da equipe médica não pretender suspender o suporte vital e, sendo assim, os exames seriam desnecessários. Afirmam ainda que "o conceito de ME deve refletir a idéia do que significa estar vivo ou morto, e não estar diretamente relacionado à necessidade de captação e doação de órgãos”.12 A Resolução 1480/97 do CFM é explícita quanto à realização de exames clínicos e complementares para o diagnóstico da $\mathrm{ME}$, independente do paciente ser ou não doador de órgãos. ${ }^{9}$ Entretanto, o fato da ME ser condição sine qua non para a remoção de órgãos vitais e a referida resolução determinar a sua notificação à CNCDO talvez induza o profissional a vincular o diagnóstico à doação.

Ao ser analisado o perfil predominante do potencial doador encontrado neste estudo, verifica-se que ele é jovem, do sexo masculino e morre por TCE. Com relação à faixa etária e sexo, os dados são similares à grande maioria dos observados no Brasil e no mundo. ${ }^{3,4,11,15}$ Uma pesquisa que buscou identificar potenciais doadores em vítimas de TCE no Instituto Médico Legal do estado de Sergipe também reitera esses achados. ${ }^{16}$

O presente estudo limitou a faixa etária aos 65 anos. Apesar de em razão da escassez de órgãos frente à demanda o limite de idade para a doação vir se estendendo cada vez mais, há trabalhos que mostram sua influência na qualidade do enxerto. Um estudo com transplantados renais de doador falecido demonstrou um declínio na função renal de $6 \mathrm{ml}$ por década de idade do doador. ${ }^{24}$

A predominância do TCE como causa do óbito confirma os achados em outros hospitais do país, ${ }^{13,15,25}$ embora a proporção deste em relação aos outros diagnósticos seja maior neste estudo, o que pode ser explicado pelo fato de o hospital onde se deu a pesquisa ser referência em trauma no estado. Todavia, nos países chamados desenvolvidos o AVE começa a aparecer como causa mais freqüente de $\mathrm{ME},{ }^{5,20}$ o que possivelmente está relacionado com a diminuição de acidentes fatais naqueles países. Um levantamento realizado no Hospital das Clínicas da Faculdade de Medicina da Universidade de São Paulo mostrou que os acidentes de trânsito representaram $44,5 \%$ de todas as causas de trauma que culminaram em ME. ${ }^{25}$

Estima-se que 10 a $15 \%$ dos pacientes que morrem em UTI apresentam quadro de $\mathrm{ME}$, enquanto que no hospital como um todo, o índice é de apenas 1 a 4\%. ${ }^{7}$ Em hospitais de trauma de uma capital do sul do Brasil, 95\% dos potenciais doadores estavam em UTI. ${ }^{26}$ Diante de tais dados, alguns pesquisadores consideraram mais apropriado calcular o número de potenciais doadores em ME a partir dos óbitos em UTI, restringindo suas pesquisas àquela unidade ${ }^{19} \mathrm{O}$ presente estudo não confirmou esses dados, uma vez que somente $25 \%$ dos potenciais doadores foram encontrados em UTI. A elevada incidência nas unidades de Urgência (41\%) e RPA $(22 \%)$ reflete a carência de vagas de terapia intensiva no hospital estudado, sendo comum a permanência de pacientes nas unidades de Urgência e RPA aguardando vaga em UTI. Considerando-se que se espera que na UTI haja maior detecção e melhor manutenção do potencial doador, ${ }^{5}$ é possível que isto explique os resultados desta pesquisa referentes ao diagnóstico de ME e ao índice de doação.

Um quarto dos potenciais doadores neste estudo apresentou sinais de ME nas primeiras horas após a admissão e metade deles nos dois primeiros dias. O tempo de permanência no hospital foi maior que três dias em $75 \%$ dos casos. Esses dados sugerem tempo suficiente para detecção e diagnóstico da ME nos padrões estabelecidos pela legislação. ${ }^{9}$

Chama a atenção neste estudo o tempo decorrido para a realização dos exames do protocolo de ME. Em 50\% dos potenciais doadores passaram-se mais de 17 horas entre o primeiro registro sugestivo de ME no prontuário e a realização do primeiro exame do protocolo. $\mathrm{O}$ uso de drogas depressoras do SNC pode ter influenciado este resultado, vez que se faz necessário aguardar sua eliminação para iniciar o protocolo. ${ }^{9,27}$

A Resolução CFM 1480/97 estabelece um período mínimo de seis horas entre os dois exames clínicos para pacientes acima de dois anos. $^{9}$ No presente estudo, $90 \%$ dos potenciais doadores tiveram intervalo superior a seis horas entre os dois exames, sendo que em metade deles, o intervalo foi maior que doze horas.

Em 75\% dos pacientes que se tornaram doadores efetivos, o tempo total desde a suspeita de $\mathrm{ME}$ até a remoção dos órgãos foi maior que 30 horas. É importante ressaltar que esse período é freqüentemente pontuado pela instabilidade das condições do potencial doador, que aumenta proporcionalmente com o passar do tempo. ${ }^{28}$ Um estudo realizado com transplantados cardíacos sugeriu que o tempo de ME do doador superior a 20 horas está relacionado a riscos no pós-operatório imediato do transplante..$^{29}$

Entre os não doadores, metade apresentou parada cardíaca irreversível mais de 70 horas após o primeiro registro sugestivo de ME, confirmando estudos anteriores, ${ }^{13,28}$ e os suportes vitais foram mantidos até o final. A Resolução CFM 1480/97 considera o "ônus psicológico e o material causado pelo prolongamento do uso de recursos extraordinários para o suporte de funções vegetativas em pacientes com parada total e irreversível da atividade encefálica”. ${ }^{9}$ O mesmo conselho, em sua Resolução 1826/07, estabelece que "é legal e ética a suspensão dos procedimentos de suportes terapêuticos quando determinada a morte encefálica em não doadores de órgãos, tecidos e partes do corpo humano para fins de transplante". ${ }^{30}$ Diante dessas considerações legais e tendo em vista o já referido déficit de leitos em UTI, é ético não completar o diagnóstico e manter tais pacientes até a parada cardíaca? Morte encefálica significa que o suporte de vida é inútil e a única razão para a sua manutenção é a doação de órgãos. ${ }^{18}$

Apenas 7\% dos potenciais doadores detectados neste estudo tornaram-se doadores efetivos. Este índice está bem abaixo dos padrões internacionais.7 Na Espanha e nos Estados Unidos o índice 
de doação está entre 40 a 60\%.3-6 Mesmo em termos de Brasil, a taxa encontrada neste estudo está aquém daquelas encontradas nas regiões Sul e Sudeste.,11,24 Um estudo que analisou o desempenho de uma OPO em São Paulo mostrou que 33\% dos potenciais doadores notificados tornaram-se doadores efetivos nos anos 1997 a 2000 e este índice aumentou para 37\% entre 2000 e 2003. Uma possibilidade apontada por aqueles autores para justificar a melhora dos índices é que atualmente, pacientes com pontuação na escala de Glasgow menor que 5, em decorrência de causas conhecidas, já começam a ser avaliados como potenciais doadores, enquanto anteriormente isto só ocorria a partir do momento em que chegavam ao nível 3 da tabela de Glasgow, quando alguns já apresentavam disfunções orgânicas, inviabilizando a realização do protocolo. ${ }^{31}$

No presente estudo, 31\% dos potenciais doadores não se tornaram doadores efetivos por recusa da família, o que está em consonância com o registrado na literatura, apesar de que padrões internacionais estabelecem como aceitáveis índices em torno de 20\%.7 A parada cardíaca irreversível foi a causa da não remoção em $29 \%$ dos potenciais doadores, o que é superior ao relatado na literatura, que é de 10 a $20 \% .^{28}$ Este resultado pode estar relacionado à demora para a constatação da $\mathrm{ME}$, bem como às dificuldades na manutenção dos potenciais doadores.

Embora a revisão de prontuários seja vista como o padrão ouro para a avaliação do número de potenciais doadores de órgãos, ${ }^{4}$ ela pode ser criticada, porque os dados são coletados a partir de registros de terceiros, e, talvez por isso, sujeitos a falhas. Neste estudo, além da coleta feita em cada prontuário do hospital, os dados foram comparados com os registros de notificação e os prontuários existentes na CNCDO.

\section{CONCLUSÕES}

A partir dos dados obtidos, conclui-se que o diagnóstico dos potenciais doadores não tem sido completado, havendo baixo índice de notificação, resultando numa taxa de doação de apenas $7 \%$. O perfil desse potencial doador foi de indivíduo jovem, do sexo masculino, cuja causa do óbito foi TCE e as principais causas da não efetivação da doação foram recusa da família e parada cardíaca irreversível.

Estudos deste tipo são importantes, tendo em vista a crescente desproporção entre o número de pacientes em lista e o número de transplantes realizados. Com base no presente estudo, sugere-se o desenvolvimento de um processo educativo dirigido aos profissionais de saúde, no sentido de esclarecer sobre o diagnóstico e a manutenção do potencial doador de órgãos, bem como a obrigatoriedade da notificação da ME. Faz-se necessária ainda a realização de novos trabalhos que busquem entender a questão da recusa familiar e que possam desenvolver estratégias junto à sociedade.

\section{AGRADECIMENTOS}

À Profa. Dra. Maria Jésia Vieira que, por suas aulas de Redação de Documentos Científicos, tornou possível este artigo.

\section{ABSTRACT}

Purposes: To estimate the potential for organ donors in an emergency reference hospital; to describe its profile; to analyze the causes for the non-donation. Methods: Quantitative, descriptive, and retrospective study using data collected from medical records of up to 65 years old patients dead due to traumatic brain injury, cerebrovascular stroke, and primary brain tumor along the years 2005 to 2007 . Results: From 645 medical records assessed, 219 presented record suggestive for brain death, and 126 had Declaration Term of Brain Death duly filled out. The median age of these potential donors was 29.5 years, prevailing male donors (77\%), victims of traumatic brain injury (68\%), and at Emergency Unit (41\%). Half of them presented signs of brain death in the first two hospitalization days, and only $7 \%$ became effective donors, being the family refusal (31\%) and irreversible cardiac arrest $(28.6 \%)$ the main cause for the non-donation. Time between the first record and the removal of organs was longer than 30 hours in $75 \%$ of donors. Conclusions: Results of the study showed a low notification and diagnosis of brain death rates resulting in a reduced amount of potential donors, and consequently, a small amount of died donors' organs and tissues. Rates of family refusal and irreversible cardiac arrest suggest the need of educational actions driven to the health professionals and the society in general.

Keywords: Brain Death; Tissue and Organ Procurement; Organ Transplantation; Medical Records.

\section{REFERÊNCIAS}

1. Brasil. Ministério da Saúde. Sistema Nacional de Transplantes [atualizado em 2007 Jun; acesso em 2008 Abr 04]. Disponível em http://www.saude.gov.br/ transplantes.

2. Cirurgia - Setenta anos. Revista comemorativa do $70^{\circ}$ aniversário da Fundação de Beneficência Hospital de Cirurgia. Aracaju;1996.

3. Pérez GS, Gutiérrez MEH, Gallardo ML, Ortega JFF, Verdú DA, Ordóñez JM. Donantes de órganos em uma Unidad de Cuidados Intensivos. Med Intensiva. 2004;28:308-15.

4. Sheehy E, Conrad SL, Brigham LE, Luskin R, Weber P, Eakin M et al. Estimating the number of potential organ donors in the United States. N Eng J Med. 2003;349:667-74.
5. Domínguez MJ, Sánchez A, Guerrero A, Gordillo A, Lozano O. Medicina intensiva, muerte encefálica y donación de órganos [presentado a XXXVII Congreso Nacional de la Sociedad Española de Medicina Intensiva, Crítica y Unidades Coronárias]. Med Intensiva. 2002;26:145-234.

6. Shafer TJ, Wagner D, Chessare J, Zampiello FA, McBride V, Perdue J. Organ Donation Breakthrough Collaborative. Increasing organ donation through system redesign. Crit Care Nurse. 2006;26:33-49.

7. Garcia VD. Por uma política de transplantes no Brasil. São Paulo: Office;2000.

8. Brasil. Presidência da República. Lei 9.434 de 04 de fevereiro de 1997.

9. Brasil. Conselho Federal de Medicina. Resolução 1.480 de 08 de agosto de 1997.

10. Brasil. Presidência da República. Decreto 2.268 de 30 de junho de 1997. 
11. Boni RC, Delmonte CA, Pestana JOM. Estimativa do número de potenciais doadores de órgãos na cidade de São Paulo em relação à situação do transplante de órgãos no Brasil. J Bras Transpl. 2002;5:59-64.

12. Lago PM, Piva J, Garcia PC, Troster E, Bousso A, Sarno MO et al. Brain death: medical management in seven Brazilian pediatric intensive care units. J Pediatr. 2007;83:133-40

13. Afonso RC, Pinheiro R, Santos-Júnior PRM, Bussolaro RA, Ferraz-Neto BH, Roza $B$ et al. Notifying potential donors: perspective of help from the intra-hospital transplantation committee. Transplant Proc. 2002;34:445-6.

14. Santos ALGA, Silva AAM, Santos RF. Estimativa do número potencial de doadores cadavéricos e da disponibilidade de órgãos e tecidos para transplantes em uma capital do nordeste do Brasil. J Bras Nefrol. 2006;28:25-30.

15. Schelemberg AM, Andrade J, Boing AF. Notificações de mortes encefálicas ocorridas na unidade de terapia intensiva do Hospital Governador Celso Ramos à Central de Notificação, Captação e Distribuição de Órgãos e Tecidos: análise do período 2003 2005. Arq Cat Med. 2007;36:30-6.

16. Alves-Júnior A, Cardoso ES, Figueiredo-Neto PB, Costa PSR, Sarasqueta DFO, Farias TWS et al. Estimativa de potenciais doadores de órgãos e tecidos no estado de Sergipe. J Bras Transpl. 2003;6:208-10.

17. Pereira WA. Manual de transplante de órgãos e tecidos. 2ed. Rio de Janeiro: Medsi; 2000.

18. Wijdicks EFM. The diagnosis of brain death. N Engl J Med. 2001;344:1215-21.

19. Senouci K, Guerrini P, Diene E, Atinault A, Claquin J, Bonnet F et al. A survey on patients admitted in severe coma: implications for brain death identification and organ donation. Intensive Care Med. 2004;30:38-44.

20. Wesslau C, Grosse K, Krüger R, Kücük O, Mauer D, Nitschke FP et al. How large is the organ donor potential in Germany? Results of analysis of data collected on decease with primary and secondary brain damage in intensive care unit from 2002 to 2005. Transplant International. 2007;20:147-55.
21. Schirmer J, Leite RF, Kian FM, Lemos MC, Carrara FSA, Silva AS et al. Legislação sobre doação de órgãos e tecidos: o que pensam os profissionais de UTI? J Bras Transpl. 2006;9:469-73.

22. Morais M, Felício HCC, Silva RCMA, Ramalho HJ, Silva RF, Abbud-Filho M. Multiorgan donation in an Organ Procurement Organization: evaluation of the causes of nondonation. Transplant Proc. 2002;34:453-5.

23. ABTO. Avaliação e consenso sobre a situação dos transplantes no Brasil. São Paulo; Limay; 1999.

24. Nyberg SL, Matas AJ, Kremers WK, Thostenson JD, Larson TS, Prieto M et al. Improved scoring system to assess adult donors for cadaver renal transplantation. Am J Transplant. 2003;3:715-21.

25. Moraes EL, Silva LBB, Glezer M, Paixão NCS, Moraes TC. Trauma e doação de órgãos e tecidos para transplante. J Bras Transpl. 2006;9:561-5.

26. Kaufmann ML. Na fronteira da emancipação e da colonização através do processo de doação de órgãos [dissertação de mestrado]. Porto Alegre: Universidade Federal do Rio Grande do Sul; 2003.

27. Brasil. Conselho Federal de Medicina. Processo Consulta 2.231/02. Parecer 07/03 aprovado em 16 de janeiro de 2003.

28. Wood KE, Becker BN, McCartney JG, D'Alessandro AM, Coursin DB. Care of the potential organ donor. N Eng J Med. 2004;351:2730-9.

29. Mejia JAC, Souza-Neto JD, Carvalho-Júnior W, Pinto-Júnior VC, Mesquita FA, Brasil HB et al. Uso do óxido nítrico inalatório no tratamento da crise hipertensiva pulmonar no pós-operatório de transplante cardíaco. Rev Bras Cir Cardiovasc. 2002;17:230-5

30. Brasil. Conselho Federal de Medicina. Resolução 1.826 de 06 de dezembro de 2007.

31. Morais M, Silva RCMA, Ramalho HJ, Silva RF, Abbud-Filho M. As Organizações de Procura de Órgãos (OPOs) são efetivas? Análise de sete anos de atividade de uma OPO brasileira. Arq Ciênc Saúde. 2001;11:225-9. 